\title{
Evaluation of the Suitability of Oil Palm Fruit Bunch Stalk Fibre for Lagging of Hot Food Containers
}

\author{
${ }^{1}$ AKABA ABDUL T. ${ }^{2}$ ADEMOH NUHU A. ${ }^{3}$ AUWAL MUHAMMED and ${ }^{4}$ ABDULLAHI IDRIS B \\ ${ }^{2,4}$ Department Of Mechanical Engineering, Federal University Of Technology, P. M. B. 65, Minna, Nigeria. \\ ${ }^{1,3}$ Department Of Mechanical Engineering \\ Kaduna Polytechnic, Kaduna \\ Nigeria
}

\begin{abstract}
Oil palm fruit bunch stalk fibres obtained after the harvest from a plantation located at Offa, Nigeria. These fibers were treated with $5 \% \mathrm{NaOH}$ aqueous solution, used to reinforce polypropylene polymer and investigated for thermal insulation of hot food carrying flasks. Treated fibres were loaded in concentrations by weights of 10\%, 20\%, 30\% and 40\% as filler into polypropylene matrix, fabricated into composites with an injection moulding machine with specimen of each formulation analysed for chemical, physical, mechanical and thermal properties including density, hardness, impact/tensile strengths, thermo-gravimetric and thermal conductivity using standard test equipment to ascertain the suitability for heat resisting application. Test equipment included Lee's disc apparatus, Perkin Elmer instrument, Instron tensile, Tinius impact and Rockwell hardness testers accessed in workshops and laboratories in Nigeria. The characterization result showed the composite as nontoxic to humans if it contacted food in the flask. The physical, mechanical and thermal properties showed that only 20\%-40\% fibre loading was beneficial after which performance declined. Mechanical properties of bunch stalk reinforced composites (density, hardness, impact and tensile strength) were adequate for the service conditions. The decomposition temperature of composites was about $4800 \mathrm{C}$ which was above 3500C-4350C for most conventional domestic insulators, 2810C for pure treated bunch stalk fibre and 4350C for polypropylene matrix. Thermal conductivity reduced from 0.11-0.23Wm-1K-1for polypropylene matrix to $0.0025 \mathrm{Wm}$ - $1 \mathrm{~K}$-1 for $40 \% 10 \mathrm{~mm}$ thick fibre loaded composite while it was $0.066 \mathrm{Wm}-1 \mathrm{~K}-1$ for $50 \mathrm{~mm}$ thick rock wool, $0.036 \mathrm{Wm}-1 \mathrm{~K}-1$ for glass fibre and $0.03 \mathrm{Wm}-1 \mathrm{~K}-1$ for polystyrene used as conventional domestic insulators. This confirmed superiority of the fruit bunch stalk reinforced composites as for thermal insulation application over common domestic insulators.
\end{abstract}

Keywords: Palm bunch stalk fibre, Composite, Thermal insulator, Food flask, Thermo gravimetric analysis.

\subsection{INTRODUCTION}

Oil palm (Elaeisguineesis.) is one of the important and abundant tree crops in southern part of Nigeria. Major products from the tree are palm wine; tapped directly from tip of the tree trunk, palm oil; processed from the mesa carp of ripped harvested fruits and palm kernel oil which is usually extracted from the fruit nut kernels. After harvest and removal of oil bearing fruits the empty biomass fiber bearing bunch thrown away at farm centres constitute environmental hazards rather than put them into possible industrial uses. Attempts to utilize the biomass wastes in several other ways like organic fertilizer in oil palm plantations, fuel for boilers and other uses are limited due to their slow bio-degeneration, difficulty in bulk packaging and transportation. These justify reasons for finding dynamic external uses for this type of agro-wastes which have some realizable potential for industrial application. This work is aimed at determining suitability of fruit bunch stalk fibre as reinforcement for thermal insulation polymer composites for use in lining hot food preservation flasks. Objectives of the study include chemical, physical and mechanical characterization of fibre of palm fruit bunch stalk, chemical treatment of same, fabrication of thermal insulator composite with treated fibre as reinforcement filler and polypropylene polymer as matrix.

This will be followed by chemical, physical, mechanical, thermo-gravimetric, thermal decomposition and conductivity analyses of the composite specimens to determine their suitability for insulating hot food flasks. The significance of the work when completed successfully would lie in the fact that part of the post harvest waste from oil palm produce will be ploughed back into wealth to generate employment opportunities; minimize hazard hitherto posed by the wastes at farm sites and provide another source of 
human friendly bio-degenerable natural fibre for industrial application as replacement to some unfriendly synthetic materials like styrofoam which causes kidney failure if heated with food. Right from Babylonia period (4000-2000BC) when composites were formed from reinforced bitumen or pitch (Sanadi et al, 1995) to era of Egypt and Mesopotamia (2500BC) when different types of river boats were constructed from bundles of papyrus reed embedded in bitumen as matrix (Lindquist et al, 2003), series of studies have been advanced for different materials for application in composites production. As synthetic fibers take several decades to decompose at much slower rate than natural fibres substantial dedicated research works have steady grown since $21^{\text {st }}$ century and is expected to continue to increase throughout next centuries to diversify the uses of natural fibre reinforced composites (Broutman and Aggarwal, 1980).

Study of thermal properties of natural fibre reinforced composite is important from view point of low cost, light weight, non toxicity, high performance and abundance in addition to the fact that composites reinforced with them offer combinations of properties that give different benefits than each individual constituent (AbdulKhalil et al, 2012). Thermal insulator made of single material, mixture of materials or composites structure has ability to reduce heat flow from one end surface to another. Their effectiveness is measured from thermal properties like conductivity which depends on the physical and chemical structures of materials. Thermal conductivity is a measure of heat energy flow through unit thickness under given temperature gradient of materials under steady state conditions guided by Fourier's law. Lower thermal conductivity of material favours its suitability for insulation purposes. Heat transfer in fibrous insulator occurs through solid fiber and still conduction, convection and radiation; implying that variation in fibre content alters the thermal conductivities. Heat convection is significantly decreased by impediments of convective current; heat radiation is gradually decreased by scattering and reemission at higher density (Aris and Hassan, 2008). Thermal insulation materials are categorized as fibrous, cellular, flakes or granule that can be used as wearing apparel, building insulation and liner for flask, furnaces or pipe. Fibrous insulator has been commonly used in industrial processes such as pipings and furnace insulations typical of which are used for cryogenics, HVAC and moderate temperatures. Its flexibility and adaptability allow it fit into common shapes found in industrial process equipment and facilities. Its lightweight allows is use for temporary insulation and modified for reuse elsewhere without much incurred costs.

In order to maximize property enhancement of individual constituent of multiple component (hybrid) composites, certain rules are applied in its formulation. Based on the rules, volume fractions are calculated from the density of every individual constituent of the composite and the targeted properties of the material that is usually dictated by its expected service conditions. The fractions are calculated from the equation stated as follows:

$\mathrm{V}_{\mathrm{i}}=\mathrm{v}_{\mathrm{i}} / \mathrm{v}_{\mathrm{j}}$

where $\mathrm{V}_{\mathrm{i}}=$ volume fraction of constituent; $\mathrm{v}_{\mathrm{i}}=$ volume of individual constituent and $\mathrm{v}_{\mathrm{j}}$. $=$ volume of total constituent which is always equal to 1 . The theoretical target density of the multi constituents composite is determined from the equation:

$$
\hat{p}_{\text {composite }}=\hat{p}_{\mathrm{i}} \mathrm{v}_{\mathrm{i}+} \mathrm{p}_{\mathrm{j}} \mathrm{v}_{\mathrm{j}+} \mathrm{p}_{\mathrm{k}} \mathrm{v}_{\mathrm{k}}=\mathrm{V}_{\mathrm{i}}+\mathrm{V}_{\mathrm{j}}+\mathrm{V}_{\mathrm{k}} \ldots \ldots \ldots \ldots(2)
$$

Where $\oint_{i}, \oint_{j}$ and $\oint_{k}$ are the densities of each of the constituent components of the composite while $V_{i}, V_{j}$ and $V_{k}$ are the volume fractions of each of the constituent components in the composite.

This rule of mixture in composite formulation may not actually be of much relevance in the proposed bi-composite formulation involving only palm fruit bunch stalk fibre as a filler and polypropylene polymer as matrix. Oil palm bunch stalk contains fibers having common physical properties like materials which are used for fibrous insulation thereby prompting its use in this work for insulator composite reinforcement. Natural fibres are basically lignocellulose in nature; consisting of helically wound cellulose micro fibrils in matrix of lignin and hemicellulose (Lundquist et al, 2003). Evolving interests in plant wood derived fibres for reinforcing thermoplastic is due to low density/price, high strength, stiffness and faster bio-degenerability (Myers et al, 1991). Moreover the mechanical properties of composites are shown to drastically improve when reinforced with natural fibers sourced from jute, sisal, pineapple, abaca, coir and plant tree fibre like oil palm by Shibata et al (2003; Ademoh and Olanipekun, 2015). Particulate reinforced composites (PRC) proposed for use in this work are composites whose reinforcement is in form of particles with all dimensions roughly equal. Particulate fillers are used to improve high temperature performance, reduce friction and shrinkage and increase wear resistance (Rana et al, 1998). Particles share load with matrix to lower extent than fiber as it improve stiffness than strength. The scope is to reinforce polypropylene with particulates generated from stalk of oil pal fruit bunch for formulation of thermal insulation composite.

\subsection{MATERIALS AND METHODS}

\subsection{Materials}

The main raw material, oil palm fruit stalk was sourced from a plantation in Offa, Kwara State of Nigeria after fruits had been harvested from bunch. Sodium hydroxide pellets to be used for chemical treatment of raw palm bunch stalk fibre, distilled water and graded polypropylene (PP) used as polymer matrix were sourced from known industrial chemical/polymer dealers in Kaduna. MFI for polypropylene matrix was $1-12 \mathrm{~g} / 10 \mathrm{~min}\left(\right.$ at $230^{\circ} \mathrm{C}$ ) with density $0.9 \mathrm{~g} / \mathrm{cc}$. Figure 1 shows section of palm tree where fruit 
bunch is obtained, figure 2 shows shredded fibre obtained from fruit bunch stalk before cutting, figure 3 shows shredded stalk fibre after cutting to pieces and figure 4 shows granulated polypropylene polymer matrix.

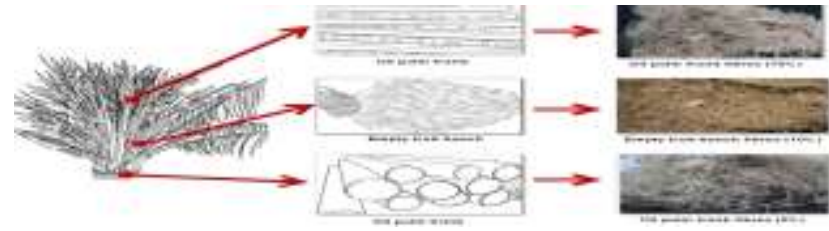

Figure 1:-Oil palm tree showing site of fruit bunch

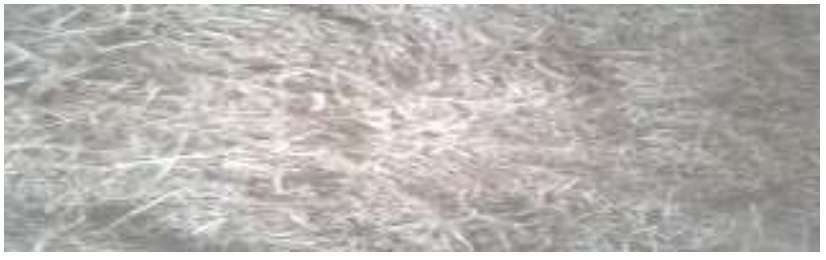

Figure 3:-Shreded bunch stalk fibre after cutting.

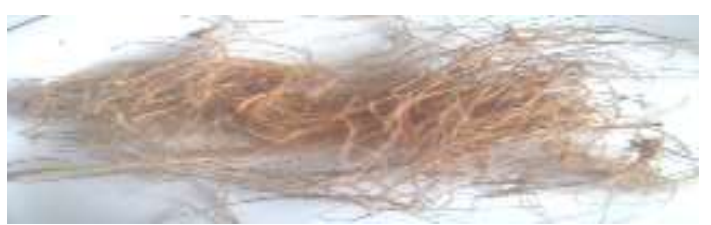

Figure 2:Shreded bunch stalk fibre before cutting

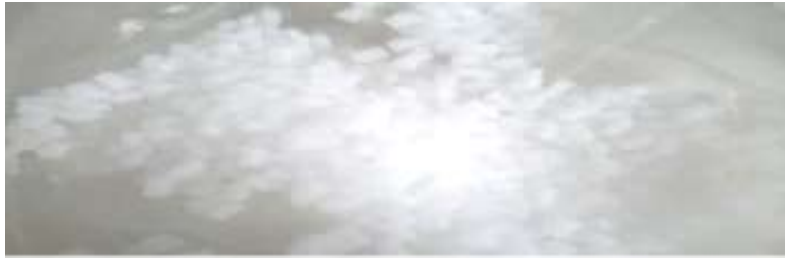

Figure 4:-Structure of granulated polypropylene

The specimen preparation equipment and those used for the analytical work included injection moulding machine (Model EC1300SX), tensile test machine (Instron Model 3360 series), impact test machine (Tinius Olsen Model 899) and hardness test machine (Rockwell B scale-HRB). Other equipment included Perkin Elmer instrument (Model 4000) and Lee's disc apparatus. The equipment were accessed/used at the workshops and laboratories of the Kaduna Polytechnic, Engineering Materials Development Institute, Akure, Federal University of Technology, Minna and Ahmadu Bello University, Zaria, Nigeria.

\subsection{Methods}

2.2.1 NaOH Chemical Treatment on Fibre:-The palm fruit stalk were reduced into shredded fibres as in figure 2 were subjected to natural retting process for a period of 4 weeks as practiced by Ademoh and Adesoji (2015) in distilled water at room temperature to condition them for $\mathrm{NaOH}$ surface modification chemical treatment. The retted fiber was manually cut into average length of $5 \mathrm{~mm}$ as shown in figure 3 to expose all fibre surfaces for penetration of aqueous $\mathrm{NaOH}$ solution during the chemical treatment.

Fiber Surface Modification and Mercerization Treatment:-Prior to chemical treatment, bundles of retted fibers were scoured in mild detergent solution at room temperature for 2 hours to remove dust and other impurities. Thereafter, the fibers were washed in distilled water and air dried for two days. Mercerization is alkali treatment process that subjected the natural fibre to interactions with fairly concentrated aqueous solution of $\mathrm{NaOH}$, a strong base in order to produce great swelling which resulted into changes in the fibre structure, morphology, thermal and mechanical properties (Bledzki and Gassan 1999). This was was carried out by immersing palm fruit bunch stalk fiber in $5 \%$ sodium hydroxide $(\mathrm{NaOH})$ for 1 hour at room temperature. This alkali solution concentration was based on previous studies which showed 5\% as the most effective for this type of fibre as observed by John et al (2008); Ademoh and Olanipekun (2015). Higher concentrations were reported to have caused delignification of natural fibre which resulted in fibre surface damage. Treated bunch stalk fibers were washed with distilled water and under continuous stream of tap water until complete removal of $\mathrm{NaOH}$ residue was achieved. Fibers were then thoroughly exposed for drying at room temperature for 24 hours to ensure minimized moisture content. Dried fibres were then stored in desiccators awaiting test and usage to avoid unneeded moisture absorption and contaminations.

2.2.2 Production of thermal insulator composite sample:-The percentage by weight of dried palm fruit bunch stalk fibre was varied in accordance with combinations polypropylene matrix as presented in table 1 to fabricate different grades thermal insulation composites. Before compositing the fibres were crushed and ground in a mill and then sieved to obtain granulate sizes of $50 \mu, 75 \mu$ and $100 \mu$ that would permit close inter-particulate contact and interaction with the polypropylene matrix for a homogeneous product.

Table 1 Compositional percentage constituents of the reinforced composite specimens

\begin{tabular}{|l|c|c|c|c|c|}
\hline Sample Code/Composition & A & B & C & D & E \\
\hline Polypropylene Content (\%) & 100 & 90 & 80 & 70 & 60 \\
\hline Palm Fruit Bunch stalk Fibre Content (\%) & 0 & 10 & 20 & 30 & 40 \\
\hline
\end{tabular}

A $200 \mathrm{~g}$ rated injection molding machine equipped with $75^{\circ} \mathrm{C}$ capacity cooling system of was used for the composite production. Each formulated sample was introduced at a time into moulding machine hopper that slid materials into the mixing chamber that is integrated with the machine. Compounding of mixture was preceded by heating each sample at temperature range $150^{\circ} \mathrm{C}-180^{\circ} \mathrm{C}$ 
followed by its suction into the die head with aid of a pump that transferred the mixture into the compounding mould. The chamber was closed and with pressure setting of 100 bars mixtures were formed and ejected from mould after each sample was allowed to cure within chamber for 20 minutes in accordance with ASTM D-3039 standard adopted by Singha et al (2008). Sections of fabricated composites were taken and prepared into specimen for tensile, impact and hardness analysis with shapes, dimensions and procedures as spelt out by ASTM D-3039. Figure 5 shows a typical sample of composite produced and ejected from moulding machine.

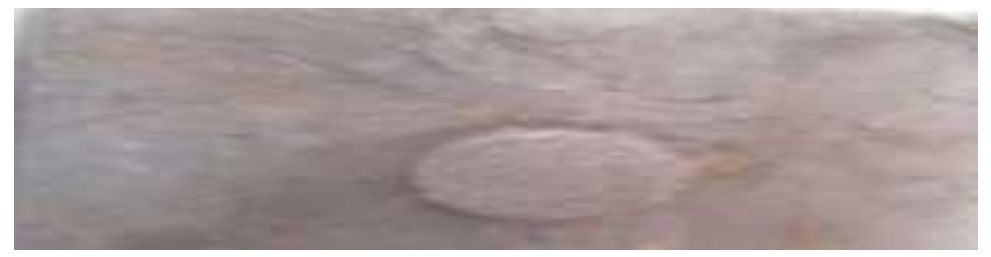

Figure 5:-Representative sample of the fabricated palm frond reinforced composite.

2.2.3 Mechanical and physical Tests on Fibres:-Tensile properties of both treated and untreated fibres were determined using the Instron universal test machine. Five typical samples of untreated and treated bunch stalk fibre were selected and tested with the results tabulated accordingly. Specimen thickness was measured with a digital micrometer screw gauge and length was measured with a standard meter rule.

To determine sample density, dried treated/untreated fibres were weighed and recorded as w. Distilled water was poured into a measuring cylinder with initial volume noted and recorded as $\mathrm{V}_{1}$, Weighed dried fibre was put into the measuring cylinder with distilled water and final volume was noted and recorded as $\mathrm{V}_{2}$ for each fibre specimen. Density was calculated using the formula in equation 1 as adopted by Abdul Khalil et al (2011). These procedures were applied to determine the density of the new composites.

$$
\frac{W}{V 2-V 1} \times 1000 \mathrm{Kg} / \mathrm{cm}^{3}
$$

2.2.4 Chemical analysis on palm fruit bunch stalk fibre:-Quantified amounts of reated and untreated fibres prepared into standard test samples and subjected to chemical analyses to determine percentage compositions of major constituent to ascertain toxicity and other inherent characteristics of the materials especially as it is proposed for application in food carrying vessels. As practiced in past related standard studies (Ververis et al, 2007), a quantity of $0.7 \mathrm{~g}$ ground stalk fibre was initially hydrolysed by boiling in a $5 \mathrm{ml}$ of $72 \% \mathrm{H}_{2} \mathrm{SO}_{4}$ solution for $4 \frac{1}{2}$ hours. The suspension remaining after the hydrolysis was filtered with the residue oven dried at $105^{\circ} \mathrm{C}$ for 24 hours, allowed to cool in desiccators, weighed and recorded as $\mathrm{W}_{1}$. The dried sample was transferred into a pre-weighed porcelain crucible, placed in a furnace, heated at $600^{\circ} \mathrm{C}$ for 5 hours, allowed to cool down, reweighed and recorded as $\mathrm{W}_{2}$. The percentage ash content in sample was determined through similar method adopted by Ademoh and Olasoji (2015). In this way, acid insoluble lignin was calculated by finding the difference in recorded weights (i. e. $\left.W_{1}-W_{2}\right)$. A $5 \mathrm{ml}$ filtrate obtained from the hydrolysis containing sugar released from cellulose and hemicellulose was measured and neutralized with $10 \%$ $\mathrm{NaOH}$, stirred and diluted to $100 \mathrm{ml}$. The glucose $\mathrm{C}_{1}$ and reducing sugar $\mathrm{C}_{2}$ concentrations in the filtrate were determined using glucose peroxidase assay kit and dinitrisalicyclic acid (DNS) methods respectively. This was done by determining rates of absorption with spectrophotometer by using the solutions derived from the procedures formulated below to determine $\mathrm{C}_{1}$ and $\mathrm{C}_{2}$ as practiced by Ververis et al (2007); Ademoh and Olasoji (2015) in closely related previous works.

$1 \mathrm{ml}$ of sample (filtrate) $+2 \mathrm{ml}$ of reagent $\mathrm{A}$

$1 \mathrm{ml}$ of glucose (Standard) $+2 \mathrm{ml}$ of reagent $\mathrm{B}$

$1 \mathrm{ml}$ of sample (filtrate) $+1 \mathrm{ml}$ of DNS $+2 \mathrm{ml}$ of $\mathrm{H}_{2} \mathrm{O}$

$1 \mathrm{ml}$ of glucose (Standard) $+1 \mathrm{ml}$ of DNS $+2 \mathrm{ml}$ of $\mathrm{H}_{2} \mathrm{O}$

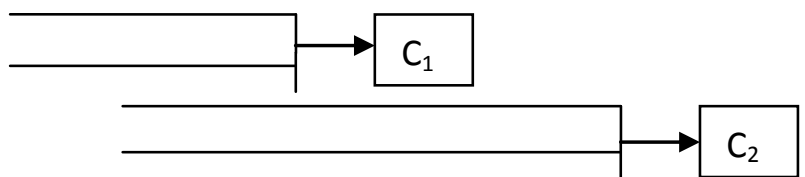

These solutions were heated up in boiling water bath for 5 minutes and absorbance was determined using the spectrophotometer at 540nm. The values of $\mathrm{C}_{1}$ and $\mathrm{C}_{2}$ were therefore determined.

Percentages of composition of cellulose and hemicellulose in starting material were calculated using the following equations that were used by Ververis et al (2007) and adopted by Ademoh and Olasoji (2015),

$\%$ w/w cellulose content $=(0.9 / 0.96) \times \mathrm{C}_{1} \times(\mathrm{V} / \mathrm{M}) \times \alpha \times 100$

$\% \mathrm{w} / \mathrm{w}$ hemicelluloses content $=(0.88 / 0.93) \times\left(\mathrm{C}_{2}-\mathrm{C}_{1}\right) \times(\mathrm{V} / \mathrm{M}) \times \alpha \times 100$; where:

0.9 is the coefficient of molecular weight ratio of the polymer and monomer hexose;

0.962 is the saccharification yield; 0.88 is molecular weight ratio of the polymer and monomer pentose;

0.93 is saccharification yield of xylane to xylose; $\mathrm{C} 1$ is the evaluated glucose concentration $(\mathrm{g} / \mathrm{L})$;

$\mathrm{C} 2$ is the evaluated reducing sugar concentration $(\mathrm{g} / \mathrm{L}) ; \mathrm{V}$ is the total volume of sugar solution $(\mathrm{L})$;

$\mathrm{M}$ is the dry weight of fibre $(\mathrm{g}) ; \alpha$ is the dilution factor $(0.05)$. 
2.2.5 Mechanical and physical characterizations of composites:-Evaluation tests were carried out on newly formulated thermal insulator composites to determine the ability to withstand physical, mechanical and thermal stresses to which each specimen would be subjected in serve. Ability of samples to preserve heat for long periods was also evaluated by determining the thermal conductivities. Preservation of heat energy within the food vessel is achieved by minimizing thermal conductivity that result to rapid heat loss from material. All the characteristics displayable by natural fibre reinforced composites depend on interfacial adhesion and bonding features as they have significant effect on strength (Ismail et. al.2004). Therefore the primary requirements for effective use of reinforcingt properties of natural fibres are good interfacial bonding or adhesion between the polypropylene polymer matrix and fiber (Singha et. al.2008). Tensile Strength:-Tensile strength test measured maximum applied load or stress that specimen withstood before breakage. It highly depended on adhesion between fiber and matrix, fiber length and fiber loading (Chai et al, 2009). As a point of importance for a major service requirement, the flask will be subjected to tension by weight of its content; tensile strength test was conducted on computerized universal machine in accordance with ASTM D-3039 standard method. A standard test sample of $10 \mathrm{~cm}$ length shown in figure 6 was clamped into each of the two $2 \mathrm{~cm}$ wide jaws of the universal strength test machine. With the initial reading set at zero, tensile stressing was done at a constant strain rate of $10 \mathrm{~mm} / \mathrm{min}$ over the gauge length of sample until failure occurred and load/extension curve was drawn from the emanating result.

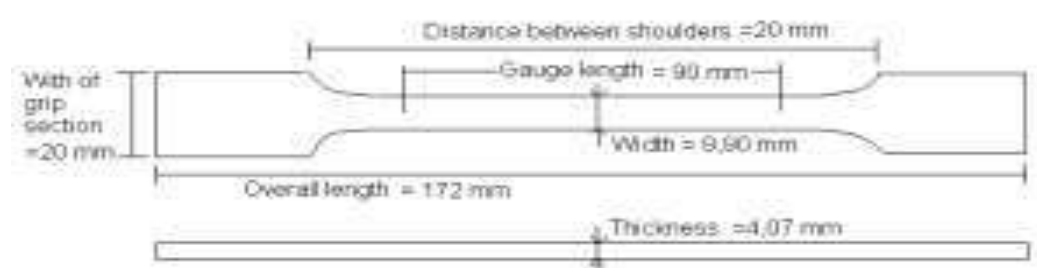

Fig.6: Tensile strength test sample.

Impact test:-The impact resistant of the palm frond reinforced insulator composite was investigated with an Izod impact tester to determine its toughness. The notched izod impact strength of the specimens was evaluated using an impactometer manufactured by Tiniusolsen, USA in accordance with ASTM-D-256 with a notch depth of $2.54 \mathrm{~mm}$ and notch angle of $45^{\circ}$ with results recorded in Joules per meter. Notches on specimens illustrated in figure 7 were made by a specimen notch maker attached to the instrument.

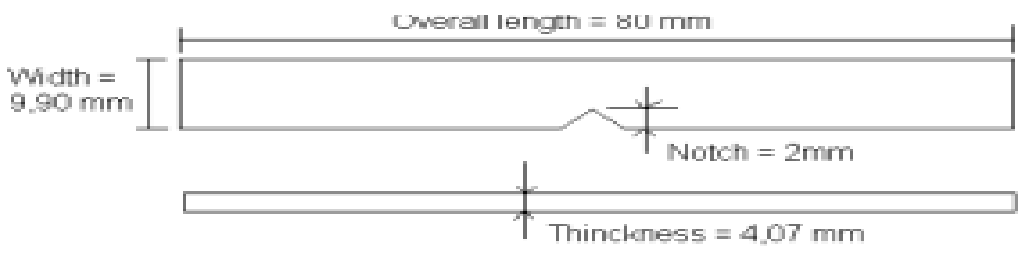

Figure 7:-Impact test sample

Hardness test:-Rockwell hardness tester (HRB- B scale) was in accordance with ASTM D-224 standard used to measure sample resistance to surface indentation, friction or wear. Samples prepared according to the ASTM procedures, the base and load bearing surfaces were made parallel to each other. As a routine check, a standard block with hardness 101.2HRB was control tested to assess the machine's status. Then, a hardened steel ball indenter was forced onto specimen surface via a minor load of $98 \mathrm{~N}$ followed by a major load of $980 \mathrm{~N}$ to make indentation as shown in figure 8 . Three separate indentations were made instantaneously across the length of each specimen. The average hardness was then calculated and noted.

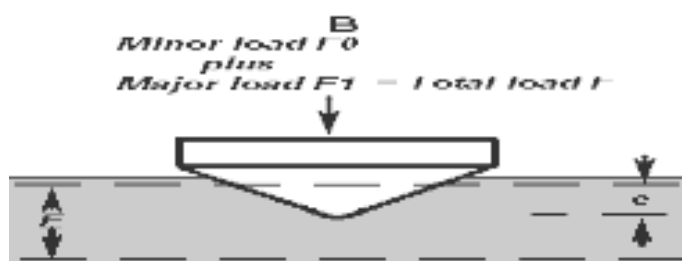

Figure 8: Rockwell hardness test sample.

Thermo-gravimetric Analysis, (TGA):-Thermo-gravimetric analysis (TGA) and differential thermal analysis (DTA) studies on newly fabricated composites samples were conducted on Perkin Elmer thermal analyzer at heating rate of $10^{\circ} \mathrm{C} / \mathrm{min}$ under nitrogen atmosphere and flow rate of $20 \mathrm{ml} / \mathrm{min}$. The TGA was used to characterize decomposition and thermal stability of samples under varieties of conditions. Change in thermal stability was basically examined by noting percentage weight loss as function of temperature. DTA compared the precise temperature difference between sample and an inert reference material as both are heated. DTG was a thermal analysis done to study the rate of change in the weight of samples against rising temperature. Results were used for simplified study of recorded weight loss due to temperature variation. All the thermal analyses were carried 
out to predict thermal behavior of samples to ascertain the basic information that foretold the thermal stability and characteristics of the formulated material.

Thermal conductivity:-Thermal conductivity analysis was carried out using the Lee's disc method. The test was conducted on composite specimens measuring $40 \mathrm{~mm}$ diameter by $10 \mathrm{~mm}$ thickness as illustrated in figure 9 with Lee's disc apparatus. A brass disc (B) was hung from a stand with the help of 3 strings. On the brass disc (B) was place a sample disc (S). A metallic disc (M) was placed on top of the sample disc $(\mathrm{S})$ and on the metallic disc, a heating chamber $(\mathrm{H})$ with facility for in and out passage of steam was created. Two holes were made in brass disc (B) and metallic disc (M) for insertion of thermometers to read temperature. Prior to test, the brass disc was weighed on a digital balance. The specimen diameter of specimen was measured with vernier caliper and thickness was measured with micrometer screw gauge.

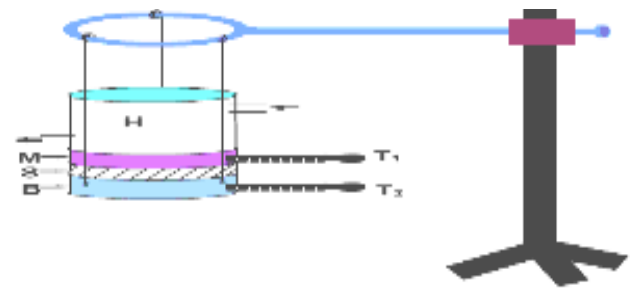

Figure 9: Lee's disc apparatus.

The heater $(\mathrm{H})$ was started by sending steam through heating chamber of apparatus. Temperatures $\mathrm{T}_{1}$ and $\mathrm{T}_{2}$ were taken at regular intervals of 5 minutes until it reached steady state. Then supply of steam was cut off and the upper metallic disc (M) and sample disc $(\mathrm{S})$ were then removed. Steam was again passed in to heat up the brass disc to $100^{\circ} \mathrm{C}$ above the steady state temperature $\mathrm{T}_{2}$. The heating chamber was thereafter to allow the cooling of the brass disc. Temperature readings were taken after every 30 seconds until it fell to about $100^{\circ} \mathrm{C}$ below steady state temperature $\mathrm{T}_{2}$. A graph placing the cooling time as abscissa and temperature of of brass disc as ordinate was developed with tangent drawn at the steady state temperature $\mathrm{T}_{2}$; the slope of which gave rate of cooling $(\mathrm{Tt})$ at steady state temperature $\mathrm{T}_{2}$. The values of $(\delta T / \delta t)_{T 2}$ was the gradient or slope of the temperature time graph at steady state and the temperature differential $\left(T_{1}-T_{2}\right)$ were determined respectively and found from raw result graph to be as follows;

$$
(\delta T / \delta t)_{T 2}=4.105 \times 10^{-30} \mathrm{C} / \mathrm{s} ; \text { and }\left(\mathrm{T}_{1}-\mathrm{T}_{2}\right)=337 \mathrm{~K}
$$

Computation of thermal conductivity of materials depended on the fact that it measured the rate at which heat flows within a body for given temperature change. Thus the rate of heat conducted through sample was given by equation 5 .

$$
Q=K A\left(T_{2}-T_{1}\right) / L \ldots \ldots \ldots \ldots \ldots \ldots \ldots \ldots \ldots \ldots \ldots \ldots(5)
$$

Where: L was the sample thickness; A was cross sectional area of sample; K was thermal conductivity; Q was rate of heat transfer and (T1-T2) was the temperature difference.

The rate of heat loss by brass disc (B) to the surrounding under steady state was given by equation 6 as.

$$
Q=m c(\delta T / \delta t) T_{2} \text {. }
$$

Where, $\mathrm{m}$ was the mass of brass disc (B); c was the specific heat of brass disc (B) and ( $\delta T / \delta t)$ was the rate of cooling at $\mathrm{T}_{2}$. A comparison of equations (5) and (6) gave rise to equation 7 as follows

$$
K=m c(\delta T / \delta t)_{T 2} / A\left(T_{1}-T_{2}\right) / L \text {. }
$$

$(\delta T / \delta t)_{T 2}$ and $\left(\mathrm{T}_{1}-\mathrm{T}_{2}\right)$ were calculated using Lee's disc apparatus through the given input value of mass of brass disc (B), specific heat of brass disc, thickness and cross sectional area of sample. Values obtained from the raw experimental result in the temperature versus time graph computations gave that:

$$
(\delta \mathrm{T} / \delta \mathrm{t})_{T 2}=4.105 \times 10^{-30} \mathrm{C} / \mathrm{s} ; \text { and }\left(T_{2}-T_{1}\right)=337 \mathrm{k},
$$

Table 2 shows weight percentage contents of palm fruit bunch stalk fibre in each composite sample, temperature and time used as parameters for computations of thermal conductivities of specimens.

Table 2: Temperature and time for computing thermal conductivities of fruit bunch stalk composites

\begin{tabular}{|l|c|c|c|c|c|c|}
\hline \multicolumn{1}{|c|}{ Measured Data Description } & \multicolumn{5}{c|}{ Values } \\
\hline Time (Minutes) & 5 & 10 & 15 & 20 & 25 & 30 \\
\hline Temp. for 20\% Fruit bunch stalk content $\left({ }^{0} \mathrm{C}\right)$ & 39.5 & 37.9 & 35.9 & 34.9 & 33.9 & 33.7 \\
\hline Temp. for 30\% Fruit bunch stalk content $\left({ }^{\circ} \mathrm{C}\right)$ & 36.3 & 33.4 & 32.8 & 30.9 & 30.1 & 30.0 \\
\hline Temp. for 40\% Fruit bunch stalk content $\left({ }^{0} \mathrm{C}\right)$ & 34.0 & 31.6 & 30.0 & 28.2 & 26.0 & 29.5 \\
\hline
\end{tabular}

Processing of thermal conductivity test data:- By reference to results generated from thermal conductivity tests the following computations were done to arrive at specific thermal properties of the composites. 
Sample thickness, $\mathrm{X}=10 \mathrm{~mm}=0.01 \mathrm{~m}$; Diameter of sample, $\mathrm{d}=40 \mathrm{~mm}=0.04 \mathrm{~m}$; Cross-sectional area of the sample, $A=\pi r^{2}=\pi \mathrm{d}^{2} / 4$; constant variables from above equations being:

Mass of brass (B) $\mathrm{M}=0.097 \mathrm{~kg}$; Cross-sectional area of sample $=1.2568 \times 10^{-3} \mathrm{~m}^{2}$;

Sample thickness $=0.01 \mathrm{~m}$; Specific heat of brass disc $=0.38 \mathrm{KJ} / \mathrm{Kg} . \mathrm{K}$

By reference to equation 1, Fourier's equation of heat transfer and from equations 2, 3, and 4 above;

$K=m c(\delta T / \delta t)_{T 2} L / A\left(T_{2}-T_{1}\right)$; Where $\mathrm{k}=$ thermal conductivity of sample

From table 2 that showed the temperature against time as obtained from the experiment the thermal conductivities (k) of the composites were calculated at different fiber constituents. To calculate the thermal conductivity K of fruit bunch stalk composite at $20 \%$ fiber loading is as follows:

$(\delta T / \delta t)_{T 2}=4.105 \times 10^{-3}{ }^{0} \mathrm{C} / \mathrm{s}$ from experimental raw result curve of temperature against time, and $\left(T_{2^{-}} T_{1}\right)=337 \mathrm{k}$, obtained from graph. These constant variables are inserted into equation 4 above to determine $\mathrm{K}$ :

$$
\mathrm{K}_{20}=\frac{\left(0.097 \times 0.395 \times 4.105 \times 10^{-3} \times 0.01\right)}{\left(1.2568 \times 10^{-3} \times 337\right)}=0.00371 \mathrm{w} / \mathrm{mk}
$$

Similarly the values of $\mathrm{K}_{30}$ and $\mathrm{K}_{40}$ were computed respectively and plotted on charts for the composites.

\subsection{RESULTS AND DISCUSSION}

3.1 Results of physical and mechanical tests on treated/untreated fruit bunch stalk fibre:-The result of the physical and mechanical properties tests on the treated and untreated oil palm fruit bunch stalk fibres ground to particulates used to reinforce the thermal insulator composite is as presented in table 3.

Table 3: Physical and Mechanical Properties of the Fibres

\begin{tabular}{|c|l|c|c|c|c|c|c|}
\hline S/N & \multicolumn{1}{|c|}{ Fibres } & $\begin{array}{c}\text { Length } \\
(\mathrm{mm})\end{array}$ & $\begin{array}{c}\text { Diameter } \\
(\mathrm{mm})\end{array}$ & $\begin{array}{c}\text { Density }(\mathrm{X} \mathrm{10}- \\
\left.{ }^{3} \mathrm{Kg} / \mathrm{cm}^{3}\right)\end{array}$ & $\begin{array}{c}\text { Tensile strength } \\
(\mathrm{Mpa})\end{array}$ & $\begin{array}{c}\text { Young Modul } \\
(\mathrm{Gpa})\end{array}$ & $\begin{array}{c}\text { Elongation at } \\
\text { break }(\%)\end{array}$ \\
\hline 1 & $\begin{array}{l}\text { Untreated stalk } \\
\text { fibre }\end{array}$ & $128-253$ & $0.12-0.27$ & 4.56 & $166.35-522.25$ & $0.501-2.765$ & $9.73-35.21$ \\
\hline 2 & $\begin{array}{l}\text { Treated fruit } \\
\text { stalk fibre }\end{array}$ & $126-252$ & $0.041-0.15$ & 1.85 & $175.24-576.34$ & $1.532-4.223$ & $7.312-11.13$ \\
\hline
\end{tabular}

In the result it is observed that there was a slight decrease in length of the fibre after treatment due to the removal of ash from both along the length and breadth of the material during modification. The diameter of treated specimen also got reduced due similar reason. The density of untreated fibre reduced by about $59.4 \%$ to give density of treated fibre. The changes in measured physical characteristics occurred because the mercerization alkali treatment subjected the vegetable fibres to interactions with fairly concentrated aqueous solution of strong base that resulted to changes in fibre structure, morphology, physical, thermal and mechanical properties (Bledzki and Gassan, 1999). It reduced weight of fibres through removal of the unwanted constituents like ash in fibre. $\mathrm{NaOH}$ treatment caused benefitial effects like moisture removal to increase strength, flexural rigidity, clearance of impurities adjoining fibre, stabilization of molecular orientation and surface modification of the plant fiber for reinforcing polymers.

It also removed lignin, hemicellulose, wax and oil which covered the surfaces of fibre (Reza, Jamaludin and Abdul Rahman, 2013). The contaminants removal caused weight reduction which lowered density and increased strength are desired properties of the material as dictated by service to which it will be subjected. The mechanical properties of untreated palm fruit bunch fibre agreed with values obtained from past works by Ademoh and Adesoji (2015); Abdul Khalil (2012). From the results, tensile strength of treated fibre improved by 5.4-10.3\% over the untreated fibre; Young's modulus of elasticity of treated fibre was increased by 50-205\%; while elongation at break point of treated fibre got reduced by $25-68 \%$ over that of untreated palm fruit bunch stalk fibre which confirm benefits of chemical treatment of raw palm fibres as concluded in past related works Ademoh and Adesoji (2015); Abdul Khalil (2012).The results also indicated the suitability of the fibre for strengthening the polymer matrix for the desired application as food carrires the require some strength to withstand the weight of the intended content.

3.2 Result of chemical analysis on palm fruit bunch stalk fibre:-Table 4 presents basic chemical constituents of the treated and untreated palm fruit bunch stalk fibre used for the composite production.

Table 4: Result chemical analysis of treated and untreated oil palm fruit bunch stalk fibre

\begin{tabular}{|c|l|c|c|c|c|}
\hline S/N & \multicolumn{1}{|c|}{ Fibre } & Cellulose (\%) & Hemicellulose (\%) & Lignin (\%) & Ash (\%) \\
\hline 1 & Untreated oil palm frond fibre & 63.12 & 21.11 & 14.35 & 2.10 \\
\hline 2 & Treated oil palm frond fibre & 82.17 & 8.56 & 7.23 & 1.32 \\
\hline
\end{tabular}


It is observed in the results presented in table 4 that cellulose concentration increased by about $30.28 \%$; hemicelluloses content was reduced by about $60 \%$; lignin was decreased by about $50 \%$ while ash content was decreased by about $37 \%$ by treating the palm bunch stalk fibre with $\mathrm{NaOH}$ chemical. The removal or reductions of most naturally accompanying undesired constituents of fibre by mercerization left it with higher cellulose content which improved the strength and lowered its hydrophilic nature thereby imparting better surface wetting properties on the material. Specifically, these were attained through the increase in cellulose with attendant decreases in hemicellulose, lignin and ash content of the treated over untreated fibres. As shown by result in the table none of the constituents of palm fruit bunch stalk fibre was found toxic or radioactive as attested to by past related work of Abdul Khalil (2012); thus confirming its suitability for use in manufacture of hot food carrying flask. This is in order with the growing medical concerns on the development and use of anticancer products which has made virtually every synthetic materials suspect as a causative or aggravative agent. As naturally derive products from plant fibres, the usual challenges of use of synthetic materials bordering on environmental degradation, renewability of raw materials, disposability after use are minimized by benefits accruable to application of this material.

3.3 Result of mechanical property test on fruit bunch stalk reinforced insulator composite:-The results in table 5 present the hardness, impact and tensile strength values obtained from the mechanical property tests on plain polypropylene polymer used as the matrix in the composite formulation.

Table 5: Properties of the experimental Polypropylene

\begin{tabular}{|c|c|c|c|c|c|}
\hline Polymer & $\begin{array}{c}\text { Tensile strength } \\
(\mathrm{MPa})\end{array}$ & $\begin{array}{c}\text { Impact strength } \\
\left(\mathrm{Jm}^{-1}\right)\end{array}$ & $\begin{array}{c}\text { Hardness } \\
(\mathrm{HRB}-\mathrm{No})\end{array}$ & $\begin{array}{c}\text { Density } \\
\left(\mathrm{Kg} / \mathrm{m}^{3}\right)\end{array}$ & $\begin{array}{c}\text { Thermal Condct.(Wm } \\
{ }^{-}\end{array}$ \\
\hline Graded PP & $25-40$ & $20-100$ & $80-100$ & $900-910$ & $0.11-0.23$ \\
\hline
\end{tabular}

Mechanical characterization of the material was done to provide a basis for evaluation of the effect of its reinforcement with oil palm fruit bunch stalk particulates to ascertain its compatibility with the material.

3.3.1 Result of impact test:-Result of notched izod impact test is shown in figure 10 for the thermal insulator composites with differing percentage contents of polypropylene and palm bunch stalk fibers.

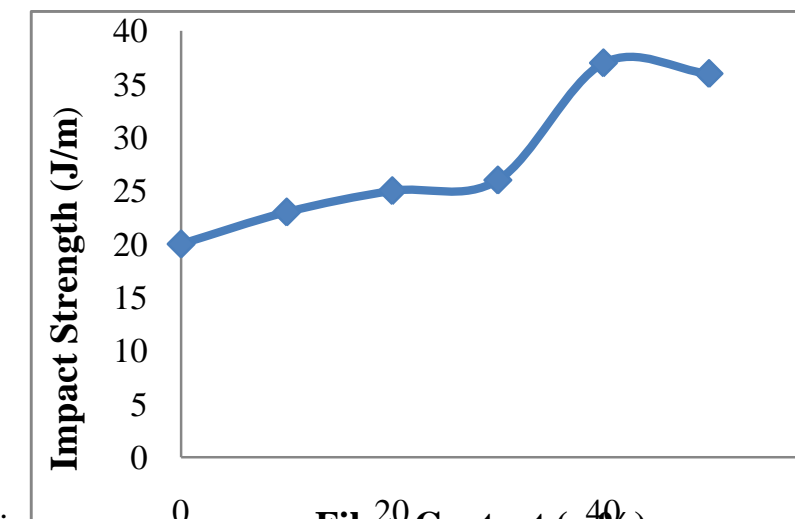

Figure 10: Impact strength against fiber content for oil palm bunch based composite

The result showed that addition of $0-30 \%$ palm fruit bunch stalk particulates to polypropylene polymer marginally improved impact strength. Below $10 \%$ and above $40 \%$ fibre contents, no significant change in impact strength of specimen occurred. From $30 \%$ fibre addition strength rapidly increased to a maximum value of $37 \mathrm{~J} / \mathrm{m}$ at $40 \%$ fibre and thereafter started to decrease. The reason was that below 30\% fibre there wasn't sufficient reinforcement filler to maximize inter-particulate reactions between matrix and filler leading to continuous increase in impact strength as fibre content increased up to optimum level of $40 \%$. A study by Plackeet et al (2003) showed that when fibre was treated, its attendant improved adhesion reaction between fiber and matrix caused considerable increase in impact resistance. At above $40 \%$ fibre concentration, the interfacial bonding between fibers and matrix was reduced due to increasing fibre to fibre contact caused by saturation. As a result less energy was absorbed during impact. Chai et al (2009) showed that adding untreated softwood fiber to reinforce polypropylene caused reduced impact strength with increased fiber content. However the result showed improved impact resistance over virgin material (without fibre). The palm stalk reinforcement increased ability of composite to withstand higher impact especially in form of weighted foods which may be loaded into the flask.

3.3.2 Result of hardness test:-The hardness of the polypropylene matrix and natural frond fiber based thermal insulator composites were investigated with the result presented in figure 11. 


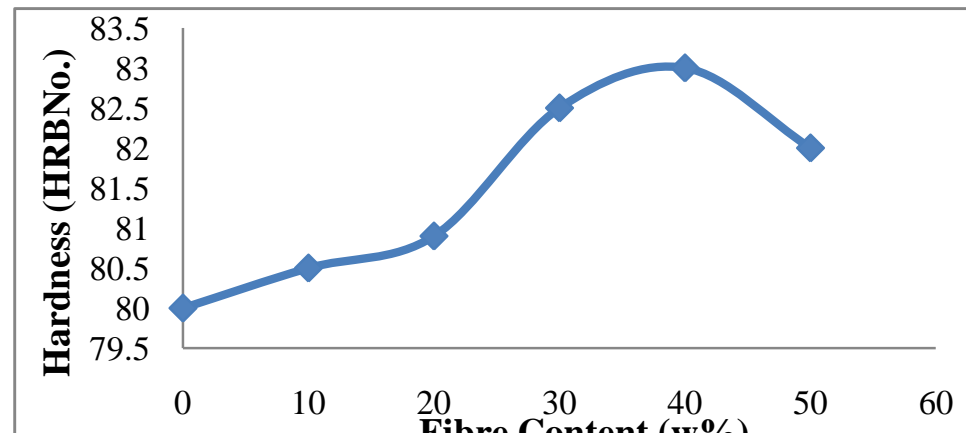

Figure 11: Hardness against fiber content for oil palm bunch based composite.

Hardness of specimens increased gradually with bunch stalk fiber content $80 \mathrm{HRB}$ No. at $0 \%$ to $81 \mathrm{HRB}$ No. at $40 \%$ fibre content in composite. It rose steeply to $83 \mathrm{HRB}$ No. at $40 \%$ stalk fibre and then dropped. The reason for this trend of result was like reasons adduced to the pattern of impact test result. Hardness was a measure of the resistance of the material to abrasion and deformation and anything that positively affected impact resistance will affect hardness of composite. As observed in impact strength test result, reduction in hardness at over $40 \%$ fibre was caused by reduction in adhesion between stalk fibre/matrix which reduced bond strength holding them together and lowered resistance to deformation of material. Hardness and impact resistance of composite are known to be affected considerably by selection of fiber reinforcement and matrix. Thus, increased hardness of composite reinforced with treated bunch stalk over matrix was due to better adhesion between fiber and matrix. According to Maryam Talimi (2011), beyond certain filler content, there was depression in hardness as due to excess fibre to fibre with lower adhesion and interfacial bond reactions. Higher filler-to-filler interaction created more voids in the composite as not all filler particles were surrounded by matrix for close bonds. Jacob et al (2009) reported that this initiated crack formation and propagation in composite and lowered strength than composite with moderate fiber loading of 20-40\%. Another reason for weakening of strength at over $40 \%$ filler is that high fiber content caused more degradation during compounding of composite due to interparticulate friction which raised temperature. More fiber composite gave higher friction causing more degradation. Natural fibers have low thermal stability, degrade and loose strength as temperature rises above $200^{\circ} \mathrm{C}$.

3,3,3 Result of tensile strength:-Tensile strength test was conducted on samples of thermal insulator composites reinforced with varied palm fruit bunch stalk filler contents with the result presented in figure 12 . In the result, tensile strength increased gradually from $25 \mathrm{mPa}$ for pure polypropylene with $0 \%$ filler to $30 \mathrm{mPa}$ at $20 \%$ bunch stalk filler content. It then steadily increased through $30 \%$ to $57.8 \mathrm{mPa}$ at $40 \%$ and then slightly dropped. Comparatively these values are lower than tensile strength of untreated (166$522 \mathrm{mPa})$ and treated $(175-576 \mathrm{mPa})$ palm fruit bunch stalk fibre as presented in table 3 .

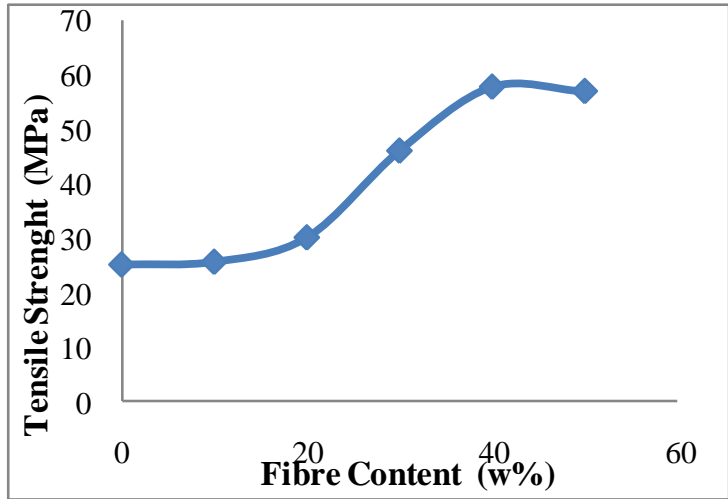

Figure 12: Tensile strength against fiber content for oil palm bunch based Composites.

The higher tensile strength of bunch stalk filler was able to raise the lower strength of matrix for better tensile properties which is desirable for the fabricated composite. Increment in tensile strength followed similar trends in impact and hardness results as it was more significant between fibre contents of $20 \%$ and $30 \%$ and reached optimum of $40 \%$ filler. This showed effectiveness in stress transmission from the matrix to the reinforcing fillers. The observed increase in tensile strength was attributable to the chemical modification treatment on the natural palm fibre as it promoted a level of chemical interaction between front particle and polypropylene matrix by way of close adhesion. Weyenberg et al (2005) reported that alkaline treatment gave up to $30 \%$ increase in tensile properties for flax fiber reinforced epoxy composite. Jacob et al (2009) also reported that composite strength/physical properties depended on the degree of interaction between polymer matrix and filler up to some limitations. It showed capability to support stress transmitted from polymer matrix was relatively improved by treatment. Sufficient tensile strength is needed for the fabricated material to adequately withstand weight of its content. 
3.3.4 Result of the density tests on the composites:-In figure 13 the result of density tests on fabricated thermal insulator composite showed that density decreased with increasing content of bunch stalk fibre due to the fact that the added filler had lower density than plain polypropylene.

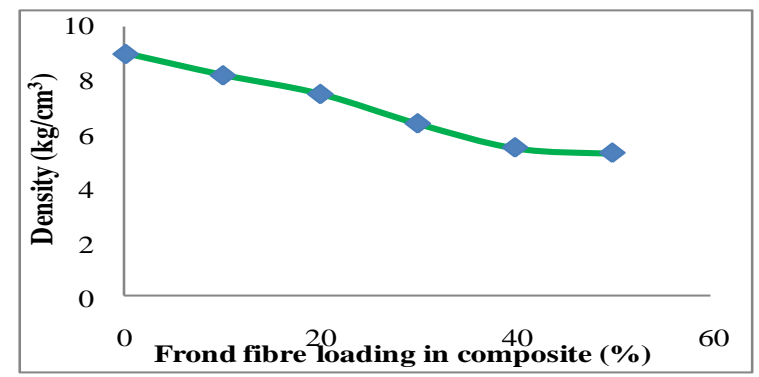

Figure 13:-Result of density tests on thermal insulator composites with varied palm bunch stalk loading.

Just as observed in past work by Ademoh and Olanipekun (2015) the density of the fabricated composite was higher than that of the palm fibre but lower than that of polypropylene matrix. Lowering of density with increased filler is desirable so as not to increase the self weight of the vessels before they are loaded with content. In comparison with densities of conventional heat insulating materials like rock wool, glass fibre and polystyrene in table below the material is suitable as the differences are not wide

3.4 Therma property analyses:-Thermogravimetric analysis (TGA) was used to investigate the effect of elevated temperature on thermal behaviour like conductivity and thermal degradation of newly fabricated composite to ascertain its heat resisting ability. Hot food flasks require chemical stability and minimal heat loss to forestall degeneration of the material or its content after elongated period of storage.

3.4.1 Thermogravimetric Analysis (TGA):-This was used to investigate possible temperature related effects on the properties of palm fruit bunch stalk reinforced composite fabricated for use as insulator paddings in food flasks. Thermal degradation monitored at programmed rate of $10^{\circ} \mathrm{C} / \mathrm{min}$ from room temperature to $600^{\circ} \mathrm{C}$ under constant flow of nitrogen gas. The onset temperature $\left(\mathrm{T}_{\mathrm{o}}\right), 50 \%$ degradation temperature $\left(\mathrm{T}_{50 \%}\right)$, peak degradation temperature $\left(\mathrm{T}_{\mathrm{P}}\right)$ and degradation temperature range $\left(\mathrm{T}_{\mathrm{R}}\right)$ were obtained from TGA and DTG curves. A plot of thermal degradation of palm fiber, pure polypropylene and the palm fruit bunch stalk based composite is as presented in figure 14. The thermograph showed that fruit bunch stalk fiber had the first peak degradation temperature of $281^{\circ} \mathrm{C}$ because the retained moisture in the fibres even after the chemical treatment and initial drying process vapourized and exited from the fibres at this elevated temperature of $70-100^{\circ} \mathrm{C}$. This is adducible to the fact that the $\mathrm{OH}$ in moisture was more active than that of inherent water in fibre as free moisture was faster to liberate. The thermographic decay may probably be due to reagent used for treatment as it had tendency to absorb moisture.

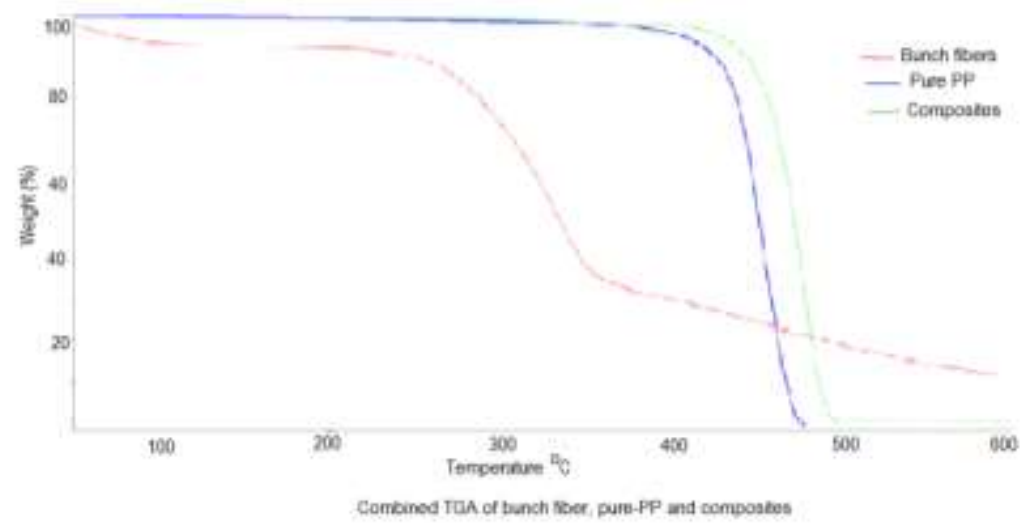

Figure 14: Combined TGA of palm bunch stalk fiber, pure-polypropylene and the new composite.

It could also be due to decomposition of cellulose, lignin and hemicellulose that gave off volatile contents as the temperature was raised (Abubakar, 2006). Pure polypropylene started degrading at temperature of $435^{\circ} \mathrm{C}$. The bunch stalk composite had onset decomposition temperature $\left(\mathrm{T}_{0}\right)$ of $456^{\circ} \mathrm{C}$. Degradation continued slowly until at about $480^{\circ} \mathrm{C}$ beyond which it rapidly progressed to a final decomposition temperature of about $500^{\circ} \mathrm{C}$. The bunch stalk fibre introduction into the matrix as a reinforcing agent was beneficial to the system as it increased the thermal stability of polypropylene from $435^{\circ} \mathrm{C}$ and that of filler from $281^{\circ} \mathrm{C}$ to $480^{\circ} \mathrm{C}$ for the reinforced composite which above the degradation temperature of most conventional domestic product insulators whose degradations are in the range $300-450^{\circ} \mathrm{C}$. This validates suitability of the newly fabricated palm fruit bunch stalk reinforced thermal insulator composite. 
3.4.2 Derivative Thermograph (DTG) of bunch stalk reinforced composite:-In figure 15 the derivative thermograph (DTG) which is a plot of derivative weight (\%/min.) against temperature for polypropylene matrix (red curve) and thermoplastic composite of varied fibre loading (blue with black dotted curves).

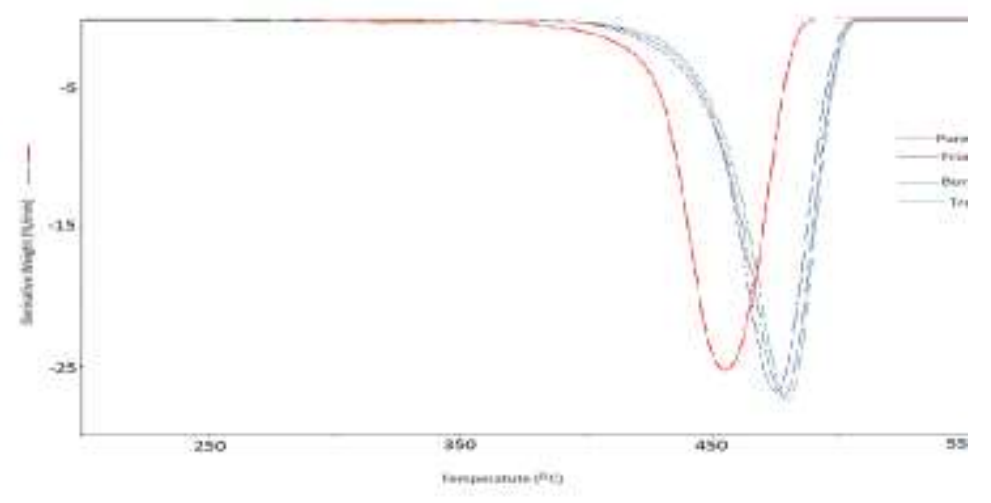

Figure 15: DTG plot for pure polypropylene and specimens of palm bunch stalk reinforced composites.

In complimentary to the TGA plot of figure 14 is the DTG presented in figure 15 . The plot showed clear evidences that depolymerization and dehydration took place between temperature ranges $91^{\circ} \mathrm{C}$ and $230^{\circ} \mathrm{C}$ which was then followed by cleavages of C-H, C-C and C-O bonds in the cellulose portion of fibre as concluded by Singh et al (2008). The initial decomposition (IDT) and final decomposition temperatures (FDT) of oil palm fruit bunch stalk were in the range $281-350^{\circ} \mathrm{C}$ in figure 14 that also showed the initial decomposition temperature of the polypropylene resin was $435^{\circ} \mathrm{C}$ and its final decomposition temperature was $480^{\circ} \mathrm{C}$. In differential thermogravimetric analysis of natural fibre, reinforced composites were shown to exhibit endothermic in addition to exothermic peaks at different temperatures when compared with the parent polymer matrix (Singha et al, 2009). On the basis on this information the magnitudes and locations of peaks in the DTA/DTG curves showed there was change in thermal behaviour of polymer matrix when reinforced with fruit bunch stalk. This further confirmed that the filler decreased the degradation process of the formulated bio-composites to buttress the achievement of the core objective of this work. It is safe to hereby conclude that the polymer composites formulated in this work is more thermally stable than either of raw polymeric polypropylene resin matrix or oil palm bunch stalk fibre when used separately. This is in agreement with Beydokhti et al (2006) who showed that most conventional synthetic materials have lower decomposition temperatures under thermal loads than natural fibre reinforced composites.

This is true because Singha et al (2008) affirmed that basically for composite to be effective load bearing system, fibre and matrix must interactively cooperate. This was done between natural palm bunch stalk fibre and polymer matrix here due to presence of good interfacial strength which in turn depended on the surface topology of the bunch stalk fibre induced by $\mathrm{NaOH}$ chemical treatment. The interface acted as the binder and transferred load between matrix and reinforcing fibre to give this good result. These available interfacial areas played major roles in determining the strength of the polymer composite because each fibre formed individual interface with matrix. Cellulose was the major component in natural palm fibre that had strong hydroxyl groups in structure which promoted hydrogen bonds within the macromolecule itself in conjunction with hydroxyl groups of polymer matrix. Indeed during fabrication of composites with oil palm fibers, thse acted as carriers of load, transferred stress from matrix along reinforcements to give the encouraging mechanical and thermal properties that were recorded in this work.

3.4.3 Thermal conductivity:-Result of thermal conductivity analyses of palm fruit bunch stalk reinforced thermo-composite carried out with Lee's disc apparatus is as in figure 16. Initial and final temperatures $\left(\mathrm{T}_{1} \& \mathrm{~T}_{2}\right)$ of each sample were measured and used to compute result in the plot that was used to evaluate performance of the composites for thermal insulation of food carrying flasks. The result in figure 16 showed that thermal conductivity decreased with increase in palm fibre content in composite as reported by Dedeepya et al (2012) in a related study who measured mechanical properties such as tensile strength, modulus and thermal conductivity of typhangustifolial fibre reinforced composite with universal strength test machine and with guarded hot plate apparatus. In the figure, increasing fruit bunch stalk $20 \%$ to $30 \%$ sharply decreased thermal conductivity of the composite specimens. However, the decrease was not significant at 30 to $35 \%$ filler content but stabilized at 35 to $40 \%$ fibre loading. 


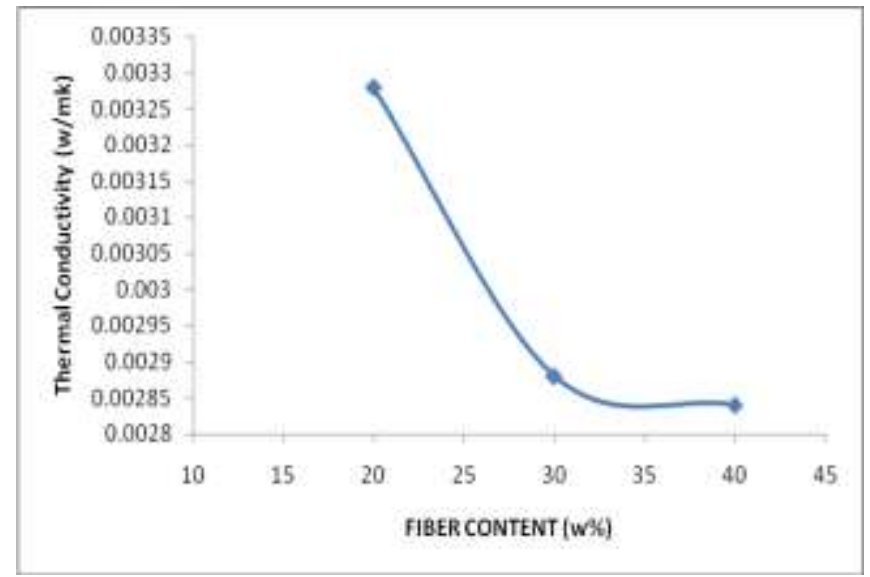

Figure 16:- Thermal conductivity (W/mk) of oil palm fruit bunch stalk reinforced composite

As the purpose of the flask is to retain the heat of its content for elongated storage period, low thermal conductivity is a critically desired property for thermal insulating materials. When the values in the figure is compared with thermal conductivities of conventional thermal insulators used for lagging domestic vessels compiled by Abubakar et al (2005), it is appreciated that oil palm fruit bunch stalk reinforced composites fabricated in this work has much lower conductivity property and are therefore classified as very suitable for this type of heat retention application. The composite has promising features for this purpose as it conductivity is lower than that of the plain polypropylene matrix that was $0.11-0.23 \mathrm{Wm}^{-1} \mathrm{~K}^{-1}$ and any of rock wool, glass fibre and polystyrene currently used for insulation of domestic heat vessels.

3.4.4 Comparative characteristics of conventional lagging materials:-Table shows two major characteristics that are used for assessing the suitability of materials for thermal insulation of domestic heat bearing containers as extracted from the work of Abubakar et al (2005).

Table 6:- Comparative thermal conductivities of selected insulation materials.

\begin{tabular}{|l|c|c|}
\hline \multicolumn{1}{|c|}{ Material } & Thermal Conductivity i. e. $\mathrm{k}\left(\mathrm{Wm}^{-1} \mathrm{~K}^{-1}\right)$ & Density $\left(\mathrm{kg} / \mathrm{m}^{-3}\right)$ \\
\hline Rock Wool & $0.016-0.026(50 \mathrm{~mm}$ thick $)$ & $9.6-32$ \\
\hline Glass Fiber & 0.036 & $64-140$ \\
\hline Polystyrene & 0.03 & $29-56$ \\
\hline Polypropylene matrix & $0.11-0.23$ & $900-910$ \\
\hline
\end{tabular}

The thermal conductivity and density of 50mm thick rock wool, fibre glass and polystyrene are selected and presented here for practical comparison and validation of the result for 10mmthick composite in this work. In accordance with Al-Nasearawi (2008) these composites could have performed better in this respect as he noted that increasing thickness causes decrease in the thermal conductivity of natural fibre reinforced composites. Comparatively densities of the material are not far below that of the composite which are within acceptable limits. The composites formulated in this work are not only suitable when compared with core characteristics of conventional insulator linings but provide some better properties combined with low cost and local availability of one of the major raw material.

\subsection{CONCLUSION}

In this research study, it was established that oil palm fruit bunch stalk reinforced thermal insulation bi-composite is not only more readily biodegradable than but also pocess properties that are comparable and even better in some instances than synthetic conventional heat resistant lining for hot food flasks. The characterized composite showed that it is non toxic and wouldn't pose food poisoning danger to humans if it gets in contact with contents of food flask. The physical, mechanical and thermal properties showed that fibre contents in composites are mostly beneficial between $20 \%$ and $40 \%$ after which depreciation occurred. The 1 properties of palm fruit bunch stalk reinforced composites including hardness, impact and tensile strength were adequate for required service conditions. Density is one of the important physical properties considered for such materials of which result here wasn't only within acceptable limit but was comparable with those of conventional thermal insulators.

Decomposition temperature of composites was above $450^{\circ} \mathrm{C}$. it is $350^{\circ} \mathrm{C}-400^{\circ} \mathrm{C}$ for most conventional domestic insulator, bunch fibre $\left(281^{0} \mathrm{C}\right)$ and polypropylene matrix $\left(435^{0} \mathrm{C}\right)$. Thermal conductivity reduced from $0.0035 \mathrm{Wm}^{-1} \mathrm{~K}^{-1}$ to $0.0025 \mathrm{Wm}^{-1} \mathrm{~K}^{-1}$ for $10 \mathrm{~mm}^{\circ}$ thick frond reinforced composites of $20 \%$ to $40 \%$ fibre respectively while values for $50 \mathrm{~mm}$ thick common conventional thermal insulators are $0.066 \mathrm{Wm}^{-1} \mathrm{~K}^{-1}$ (rockwool), $0.036 \mathrm{Wm}^{-1} \mathrm{~K}^{-1}$ (glass fibre), $0.03 \mathrm{Wm}^{-1} \mathrm{~K}^{-1}$ (polystyrene) and $0.11-0.23 \mathrm{Wm} \mathrm{K}^{-1}$ (polypropylene). Based on these results it is hereby recommended that this work should be replicated to discover the potential of other natural fibre like frond stalk and fruit fibre for related and other industrial applications. Also optimization of fiber content in polypropylene/other polymer matrix for enhanced mechanical and thermal properties; incorporation of coupling agents to 
optimize interface reactions between the fiber and matrix in order to improve mechanical and thermal properties of composites and optimization of fiber properties using other modification agents to discover better ones for chemical modification of oil palm fiber for improved performance are extensions to this work that can be considered for future study series.

\section{REFERENCES}

1. Abdul Khalil, H.P.S., Jawaid, M., Hassan, A., Paridah, M.T. and Zaidon, A., (2012). Oil Palm Biomass

2. Fibres and Recent Advancement in Oil Palm Biomass Fibres Based Hybrid Biocomposites. In Tech Jounal. (8):187-220.

3. Abdul Khalil, H. P. S., Jawaid M., and Abu Bakar A., 2011. Woven Hybrid Composites:Water

4. Absorption and Thickness Swelling Behaviors. BioResources 6(2), 1043 - 1052.

5. Abu Bakar, A., Hariharan and Abdul Khalil H. P. S. .(2005). Lignocellulose-based hybrid bilayer 1

6. aminate composite: Part I - Studies on tensile and impact behavior of oil palm fiber-glass fiber-reinforced epoxy resin. Journal of Composite Materials 39 (8):663-684.

7. Abu Bakar A. (2006)."Mechanical, thermal and processing properties of oil palm empty fruit bunch-

8. filled Imp modified unplasticised poly (vinyl chloride) composite Ph. D Thesis, Universiti Teknologi Malaysia (UTM), Johor, Malaysia,

9. Ademoh N. A. and Olanipekun C. O (2015):-“Characterization of selected bio-mass fibres of elaeis

10. guineensis for industrial applications”. Journal of Basic and Applied Research International Knowledge Press, UK. Vol. 10 (2); Pp. 66 - 78 ISSN 2395 - 3438 (print); 2395 - 3446

11. Ademoh N. A. and Olasoji C. O (2015):-"Production of motorcycle anti-crash helmet shell from

12. composite reinforced with male flower bunch stalk fibre of elaeis guineensis”. American Journal of Materials Engineering and Technology. Vol. 3 (2): Pp.27 - 34. (C Science and Education Publishing; DOI: 10.12691/materials-3-22 .

13. Al-Nasearawi M. A. N. (2008). "Palm leaf as thermal insulation material". Ibn Al-Haytham Journal of

14. Pure and Applied Science, 21 (2), 44-53

15. Aris M.S, and Hassan, S., (2008) Development of thermal Insulation Material from oil palm waste.

16. Bledzki, A. K., and .Gassan.J( 1999). “Composites reinforced with cellulose based fibres"..Progress in

17. Polymer Science (Oxford) 24 (2):221-274.

18. Beydokhti K K, Behravesh A. H, Taher A, (2006) An experimental study on mechanical and

19. microstructural .properties of microcellular foams of ABS composites, Iran Polym J, 15, 555-567,76

20. Chai, L. L.,.Zakaria S, Chia C. H., Nabihah S., and Rasid R..(2009). Physico-mechanical properties of PF

21. composite board from EFB fibres using liquefaction technique.Iranian Polymer Journal (English Edition) 18 (11):917923.

22. Ismail H, Mohamad Z, Bakar AA, (2004).The effect of dynamic vulcanization on properties of rice husk

23. powder filled polystyrene/styrene butadiene rubber blends, Iran Polym J, 13, 11-19,

24. Jacob, Maya; ,Sabu Thomas, and. Varughese K. T (2007). Biodegradability and Aging Studies of hybrid

25. biofiber reinforced natural rubber biocomposites. Journal of Biobased Materials and Bioenergy 1:118-126.

26. John, M. J., and Thomas S..(2008). Biofibres and biocomposites.Carbohydrate Polymers 71(3):343-364.

27. Khalil, H. P. S. Abdul, M. Siti Alwani, R. Ridzuan, H. Kamarudin, and A. Khairul (2008). "Chemical

28. composition, morphological characteristics and cell wall structure" Malaysian oil palm fibers. Polymer-Plastics Technology and Engineering 47 (3):273 - 280.

29. Maryam Talimi (2011). Characterization of Natural Fiber Reinforced Biodegradation Composites.

30. Mondadori N.M.L., Nunes R.C.R., ZatteraA.j.,Oliveira R.V.B. and Canto L.B., (2008)..”Effects of

31. additives on mechanical and thermal properties of plywood and sisal wood composites . Journal of Applied Polymer Science, 109(2):157.

32. Plackett D. (2003), "Biodegradable composites based on L-polylactide and jute fibres" omposites

33. Science and Technology, vol. 63, pp. 1287-1296.

34. Reza Mahjoub, Jamaludin BinMohamad Yatim, and Abdul RahmanMohd Sam. A Review of Structural

35. Performance of Oil Palm Empty Fruit Bunch Fiber in Polymer Composites, Civil Engineering Faculty, Universiti Teknologi Malaysia, P.O. Box 81310, Skudai, Johor Bahru, Malaysi, Hindawi Publishing Corporation Advances in Materials Science and Engineering Volume 2013, Article ID 415359, 9 pages

36. Shehu U, Audu H. I, Nwamara M. A, Ade-Ajayi A. F, Shittu U. M, M. T. Isa. (2014). "Natural Fibre As

37. Reinforcement For Polymers". A Review, Petrochemical and Allied Department. Polymer Division National Research Institute for Chemical Technology, Zaria, P.M.B 1052, Department of Chemical Engineering, Ahmadu Bello University, Zaria.

38. Singha AS, Thakur VK, (2008). Fabrication of HibiscusSabdariffafibre reinforced polymer composites,

39. Iran Polym Journal, 17, 541-554,

40. Tiesong L., Dechang J., Peigang H., Merirong W. and Defu L., (2008).Application of composite 
41. science engineering,Shanghai,18-19.

42. Van de weyenberg (2005) Chemical treatment natural fibers.Composites Science and Technology 62

43. (3):339-353.

44. Ververis C., Georghiou K., Danielidis D., Hatzinikolaou D.G., Santas P., Santas R., Corleti V., (2007).

45. Cellulose, hemicelluloses, lignin and ash content of some organic materials and their suitability for use as paper pulp supplements. Bioresource Technology (98) 296-301.

46. Warner S.B. (1995). Fiber science, Prentice Hall, Eagle Wood Cliffs, New Jersey, 23-27. 104 